\title{
Magnetic activity of cool stars in the Hertzsprung-Russell diagram
}

\author{
J. H. M. M. Schmitt \\ Hamburger Sternwarte, 21029 Hamburg, Gojenbergsweg 112, Germany \\ email: jschmitt@hs.uni-hamburg.de
}

\begin{abstract}
.
I review the X-ray emission from cool stars with outer convection zones in comparison to the Sun with a focus on the properties of low-activity stars. I present the recent results of long-term $\mathrm{X}$-ray monitoring which demonstrate the existence of X-ray cycles on stars with known calcium cycles. The evidence of a minimum stellar X-ray flux is presented and arguments are put forward for the view that the Sun in its extended minimum between 2008 - 2009 behaved very much like a Maunder-minimum Sun.
\end{abstract}

Keywords. Sun: activity, stars: activity, stars: coronae, X-rays: stars

\section{Introduction}

The periodic change in the frequency of sunspot appearance on the solar surface was the first observational evidence of the magnetic activity cycle of the Sun. As is well known, the frequency of sunspot occurrence on the solar surface varies with a period of approximately 11 years. After its discovery by the German amateur astronomer G. Schwabe in 1843, systematic sunspot observations have been carried out until now, and from historical records the sunspot appearance frequency can be reconstructed back to the first decade of the $17^{\text {th }}$ century, when Galileo and Schreiner made their first telescopic sunspot observations. A sunspot frequency reconstruction going back to 1610 is shown in Fig. 1, rendering the solar sunspot record probably the longest available astronomical "light" curve. Obviously, the data quality of these sunspot observations is very much non-uniform, I do draw attention to the period between $\approx 1645$ to 1715 , the so-called Maunder minimum, named after E.W. Maunder, who, contemporaneously with G. Spörer, first identified this period as one with low sun spot numbers. Long considered an observational bias due to poor data coverage, Eddy (1976) convincingly demonstrated that "this 70-year period was indeed a time when solar activity all but stopped".

Modern multi-wavelength observations have shown the solar sunspot cycle to manifest itself in almost all spectral bands, in particular, these modern observations have shown that the observed cycle signatures are strongest and most readily apparent in coronal emission features. The coronal energy loss occurs predominantly at EUV and X-ray wavelengths, and in the X-ray range the Sun and its cycle look entirely different when compared to the optical band. A complete coverage of an activity cycle of the Sun has now been obtained by both the YOHKOH and SOHO satellites. As an example I show (in Fig. 2) the changing coronal appearance of the Sun in the emission line of Fe XV at $284 \AA$ as measured by SOHO, which has been monitoring the solar EUV output since 1996. An inspection of the SOHO images shows a very profound change in the appearance of the EUV and X-ray Sun between solar minimum and solar maximum. The precise amplitudes of the solar X-ray and EUV variability is subject to some debate, and does in fact depend on the exact wavelength range considered. At harder X-rays, 
as measured for example by the GOES satellites, one finds variability amplitudes of two orders of magnitudes and more, while at softer wavelengths the variability amplitudes become smaller. Unfortunately, at soft X-ray wavelengths, where the XMM-Newton and Chandra observatories register the X-ray emission from other solar-like stars, no long term solar monitoring is available (yet).

A ground based proxy indicator of the solar soft X-ray emission is the so-called green coronal line at $5303 \AA$, which is produced by ions of Fe XIV, i.e., from plasma at temperatures very close to those ions responsible for the emission seen at EUV wavelengths in Fig.2. Historically, the green corona line has been one of the "coronium" lines, whose correct identification with highly ionized iron (rather than "coronium") led to the surprising realization that the corona of the Sun is actually much hotter than its photosphere (see discussion by Edlén 1945). Emission from this line has been monitored since the 1930es (see the solar green line "light" curve in Fig. 3), and the green line emission shows variability amplitudes of the factor 8 - 10 between solar maximum and solar minimum.

In this context it is important to consider what the Sun would look like as a spatially unresolved star. While sunspots can be - more or less - easily detected on the solar surface in spatially resolved observations (see, however, Svalgaard 2012), their effects on the total solar irradiance are quite small. While the sunspot cycle is clearly very obvious in Fig. 1-3, in total solar irradiance (TSI) the sunspot cycle is also clear but less obvious and of much smaller amplitude at a level of $0.1 \%$. Successful TSI measurements only became available since the late $1970 \mathrm{es,}$ when spaceborne radiometers were first put into operation. Since then almost three complete solar cycles have been covered; an example of the total solar irradiance vs. time is shown in Fig. 4. Somewhat surprisingly, the total solar irradiance turned out to be larger at sunspot maximum when more sunspots appear on the solar surface (at least on average) than at solar minimum. It is, however, also clear that the TSI variability is much larger when the Sun is at cycle maximum. In fact, the passage of large sunspots across the solar surface does lead to noticeable dips in the range of a few tenth of percent at maximum, with the largest so far observed depression caused by sun spots was on the order of $0.3 \%$ observed in September 2003; at those times the TSI of the maximum Sun is actually below the TSI of the minimum Sun. In ground based observations of stars such variations in output could in principle be detected but only with some difficulties.

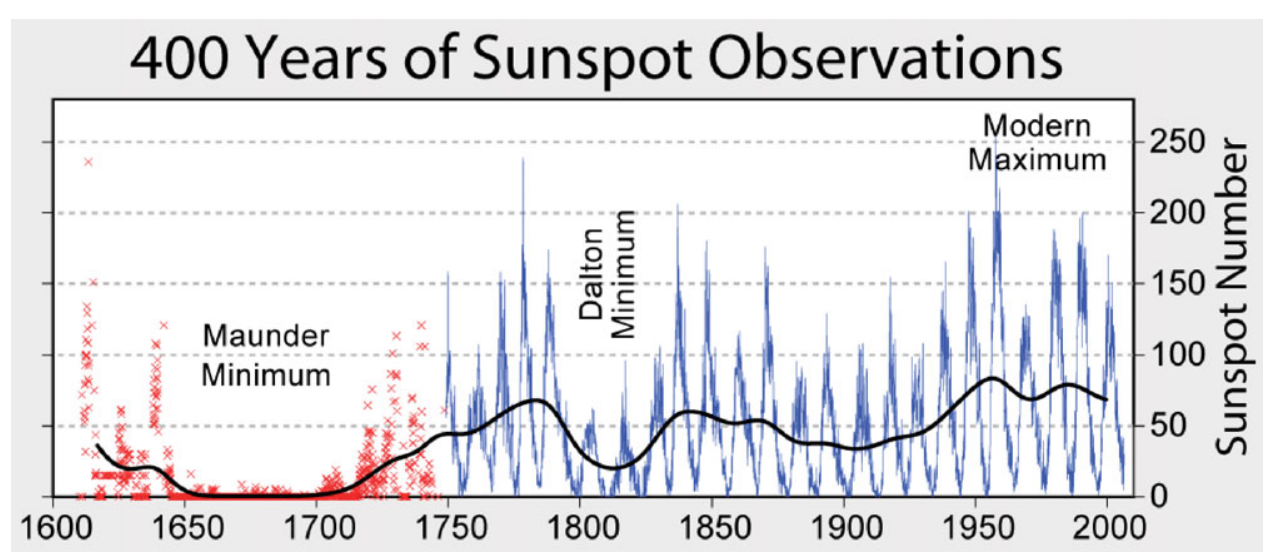

Figure 1. Sunspot number as function of time for the last four centuries (image created by Robert A. Rohde / Global Warming Art, taken from "http://www.globalwarmingart.com/ wiki/File:SunspotNumbers.png". For a colour figure, see the online version of this paper. 


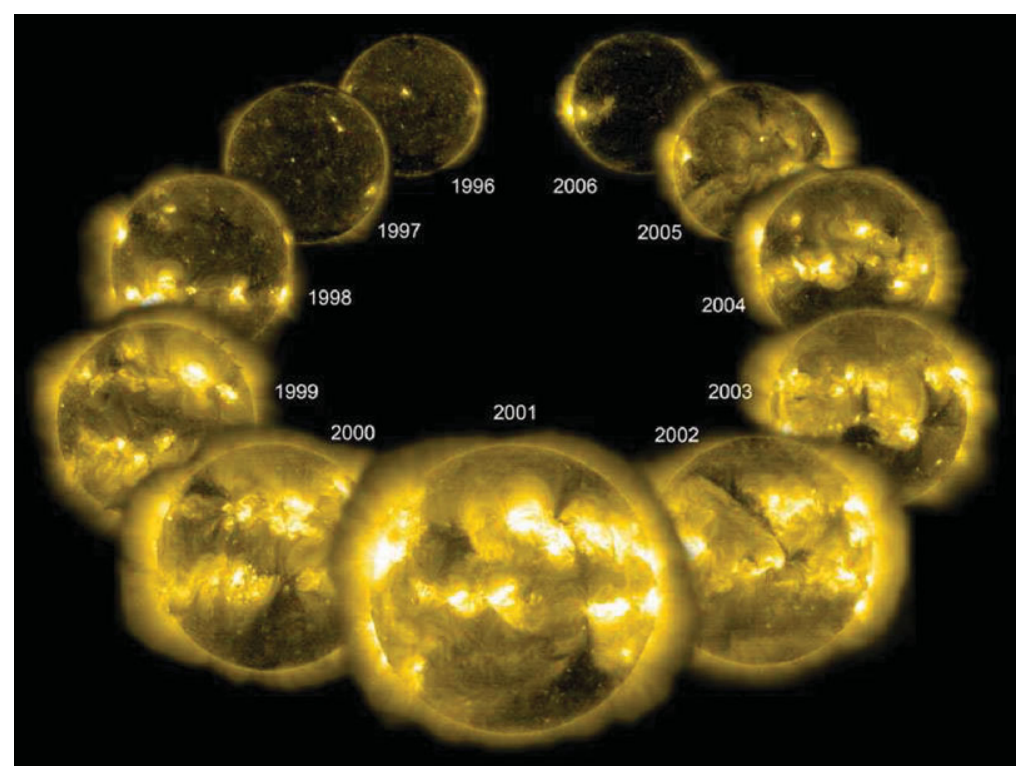

Figure 2. The changing face of the Sun as apparent in its EUV emission from Fe XV at $284 \AA$; Image credit: SOHO - EIT Consortium, ESA, NASA. For a colour figure, see the online version of this paper.

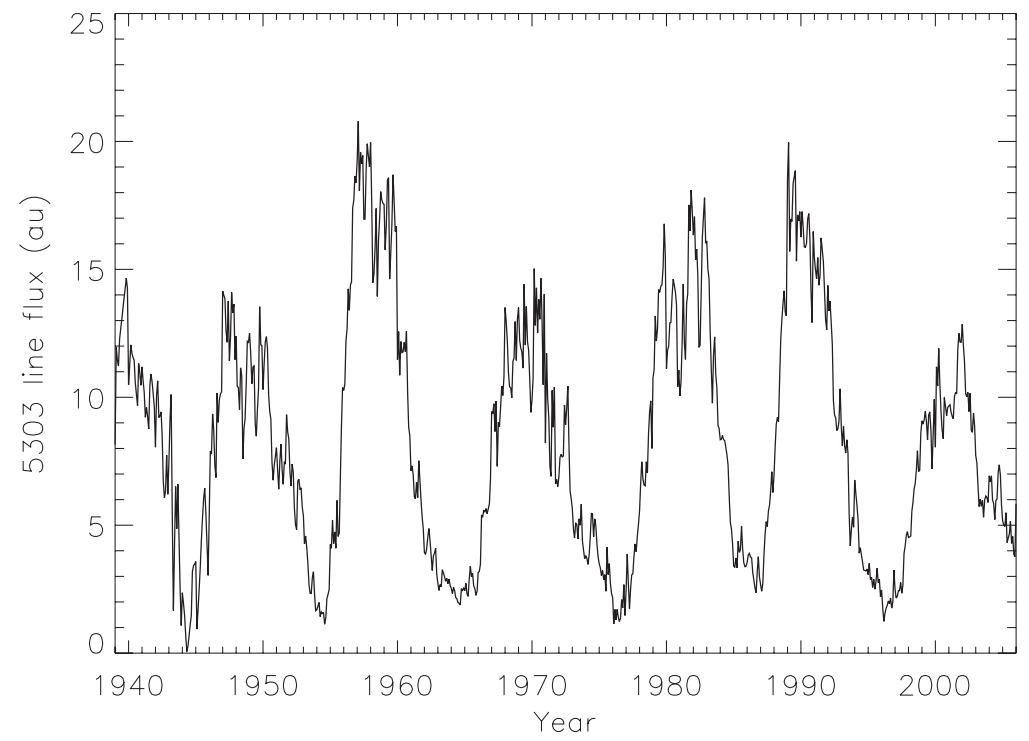

Figure 3. 60 years of variation in green line intensity (in a.u.); data source "ngdc.noaa.gov/stp/solar-data/solar-corona/index/Lomnicky/"

What makes X-ray emission particularly interesting as a physical diagnostic appears to be a correlation established between X-ray luminosity and magnetic flux. The close association of coronal X-ray emission with magnetic fields has been known, ever since spatially resolved X-ray images of the Sun had become available. By studying quiet and active Sun features as well as active stars, Pevtsov et al. (2003) were able to establish 


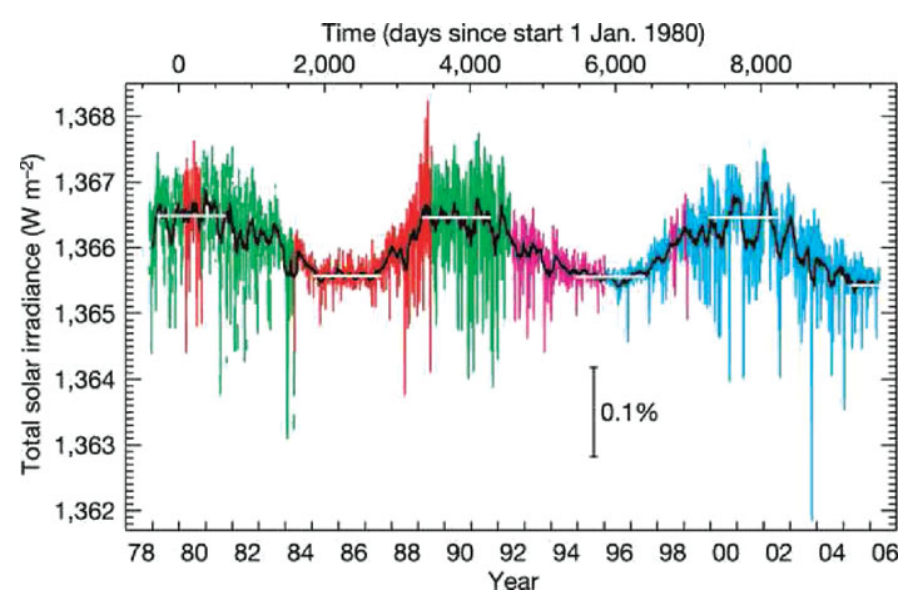

Figure 4. Total solar irradiance vs. time (taken from Foukal et al. 2006). For a colour figure, see the online version of this paper.

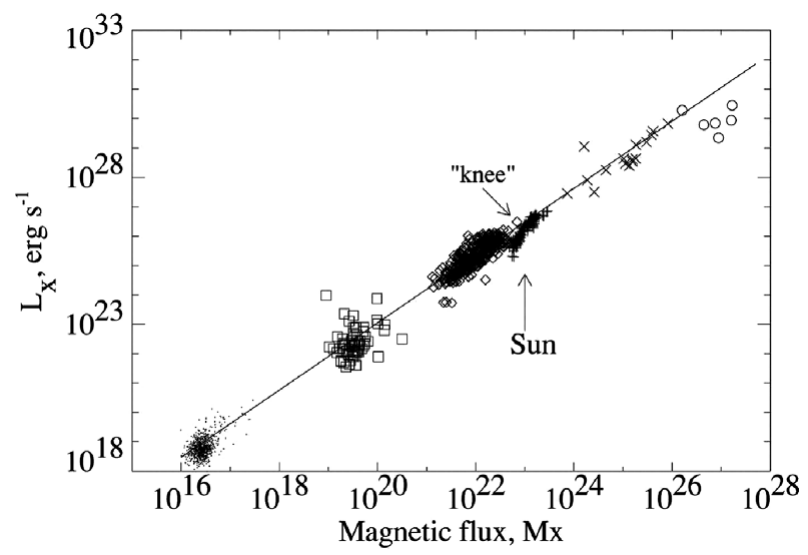

Figure 5. Correlation between X-ray luminosity and magnetic flux derived by Pevtsov et al. (2003)

an almost linear relationship between observed X-ray luminosity and magnetic flux that extends over 12 orders of magnitude, reproduced in Fig. 5. Thus, empirically X-ray emission appears to be a good proxy for magnetic fields or more precisely magnetic flux, a quantity that is very difficult to measure otherwise. Therefore the X-ray emission from other stars is used as a proxy for magnetic activity on those stars, although in many cases such magnetic fields have not been or cannot be measured directly.

\section{X-ray emission from solar-like stars}

Observations carried out with the Einstein Observatory between 1979 - 1983 were the first to demonstrate the wide-spread occurrence of X-ray emission from main sequence stars with outer convection zones (Vaiana et al. 1981). In particular, it appeared that that onset of outer convection as predicted by stellar structure theory is reflected by a rather vigorous onset of X-ray activity. This issue was studied by Schmitt et al. (1985), who found a large increase in detection rate when going from stars with $B-V \sim 0.2$ to stars with $B-V \sim 0.5$. Later the large stellar samples available from ROSAT observations allowed a precise delineation of this so-called "onset of convection". Schröder \& Schmitt 
(2007) studied the detection statistics among bright stars of spectral type B and A (i.e., stars without outer convection zones) to stars of spectral type F5 (i.e., stars with convection zones; cf., Fig.6), using the complete data set from the ROSAT all-sky survey. While there is obviously a "bottom" of about $15 \%$ of X-ray detections among A-type stars, the precise nature of which is subject of some debate, there is also a sharp increase in X-ray detections going from spectral type A7 to F2, suggesting that stars with outer convection zones find it easier to produce X-ray emitting coronae. This finding is of course in line with the idea that magnetic dynamos operate in those convection zones and it is the magnetic fields generated by these dynamos which eventually cause the observed X-ray emission (see Fig.5).
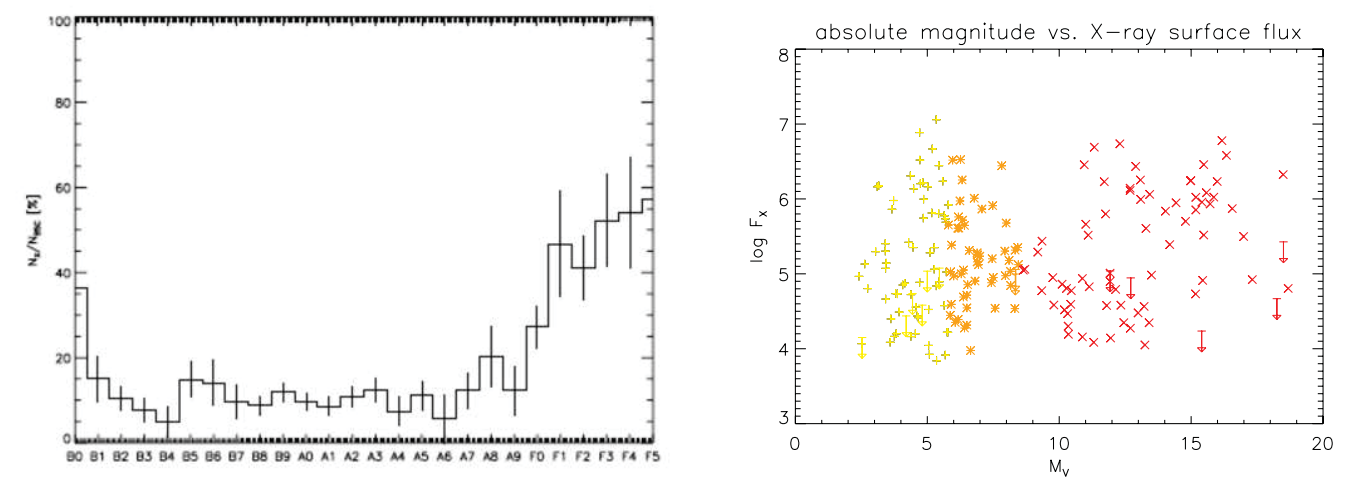

Figure 6. Left panel: detection rate of bright stars in the ROSAT all-sky survey vs. spectral type (taken from Schröder \& Schmitt 2007). Right panel: X-ray surface flux of main sequence stars vs. absolute magnitude $M_{V}$ (taken from Schmitt 1997). For a colour figure, see the online version of this paper.

What was left open by the Einstein Observatory observations was the question whether the X-ray emission for stars with outer convection was ubiquitous, i.e., whether X-ray emission is really found for all stars or whether there might possibly exist X-ray dark cool stars. To answer this question a far more systematic and, more importantly, complete study of X-ray emission from cool stars was required. To this end Schmitt (1997) investigated complete volume-limited samples of solar-like $\mathrm{F}$ and $\mathrm{G}$ dwarfs within $13 \mathrm{pc}$, $\mathrm{K}$ dwarfs within $10 \mathrm{pc}$ and $\mathrm{M}$ dwarfs within $7 \mathrm{pc}$ using data from both the ROSAT allsky survey and ROSAT pointed observations. These samples are statistically meaningful and truly complete in the sense that all stars known within this volume were observed with sufficient sensitivity to detect solar-like X-ray emission levels (and even well below), while the coverage of the low-luminosity end of the X-ray luminosity distribution function had been rather limited with Einstein Observatory data. In Fig. 6 the results of Schmitt (1997) are plotted in the form of mean X-ray surface flux $F_{X}$ vs. absolute magnitude $M_{V}$ for a complete sample of nearby stars. As is clear from Fig. 6, none of the stars with colors $M_{V}<5$ could be detected, but X-ray emission from all F-type stars was detected. Specifically, the detection rate for F-type stars is $100 \%$, the detection rate for G-stars is more than $85 \%$, with all the upper limits resulting from stars for which only survey data (and no more sensitive pointing data) were available. The conclusion from these studies must then be that coronal formation is indeed a universal phenomenon on solar-like stars. A corona containing hot plasma is always formed at the interface between a turbulent outer convection zone and space. Truly X-ray dark solar-like (main sequence) stars do 
not exist at least within the immediate solar environment and must be very rare if they exist at all.

Another salient feature in Fig.6 is the existence of a rather well defined lower envelope $F_{X, \text { lim }}$ to the observed mean X-ray surface flux distribution. The apparent cutoff at surface fluxes of $F_{X, l i m} \approx 10^{4} \mathrm{erg} / \mathrm{cm}^{2} / \mathrm{sec}$ (in the ROSAT pass band of $0.1-2.4 \mathrm{keV}$ ) is not a question of lack of sensitivity since the non-detected A-type stars do indeed have upper limits well below $F_{X, l i m}$. Because of the completeness of the samples both for the $\mathrm{F}$ and $\mathrm{G}$ stars as well as the $\mathrm{K}$ and $\mathrm{M}$ stars, one can state that among cool dwarfs stars with X-ray surface fluxes below $F_{X, \text { lim }}$ do not exist (at least in the considered volumes of space). It is interesting to compare this lower limit to the $\mathrm{X}$-ray surface flux from solar emission features. Obviously, the solar minimum surface flux is at least similar if not identical to the minimum surface flux, and Fig. 6 suggests that the stars observed at their minimum flux levels should be interpreted as stars without any active regions present on their surfaces; a further discussion of this issue is given in Section 5.

\section{X-ray cycles on other stars}

Having established the universal character of stellar coronae around cool stars, the question arises to what extent are stellar coronae similar to the solar corona and to what extent are they different. In particular, the question arises whether other stars follow the same variability pattern in soft X-rays as the Sun. Earlier studies of X-ray emission from young stars in the Hyades cluster (Stern et al. 2003) showed a relatively good correlation between the X-ray fluxes measured at two different epochs (i.e., the Einstein Observatory data taken between 1979-1983 and the ROSAT survey observations taken in 1990-1991) separated by almost a decade, suggesting that the influence of cyclic variability must be relatively modest for this group of stars. Also, a comparison of the X-ray fluxes of stars, whose cyclic properties were known from the Mount Wilson HK program, showed that stars with calcium cycles tend to show less X-ray activity than stars with irregular calcium variability (Hempelmann et al. 1996).

With ROSAT the first systematic longterm monitoring program for time variable stellar X-ray emission was initiated in the 1990s (cf., Hempelmann et al. 2003). While the Sun is essentially monitored continuously by a small armada of spacecraft, such extensive $\mathrm{X}$-ray monitoring is not feasible in a stellar context. First of all, for essentially all X-ray satellites there are viewing restrictions for any given direction, implying that a given source (star) can only be observed within some viewing interval typically lasting 4 to 8 weeks. And second, monitoring observations of stars are in competition with all other $\mathrm{X}$-ray sources studied by any given X-ray observatory, and devoting megaseconds to such programs is simply not warranted. Typical stellar X-ray monitoring programs therefore consist of individual pointings at a given star, usually separated by 6 months. With ROSAT, the monitoring of the visual binary $61 \mathrm{Cyg} \mathrm{A}$ and $\mathrm{B}$ was begun (data available from 1992 to 1998), later, with XMM-Newton the monitoring of 61 Cyg A/B has been continued (since 2002) and three more stars, $\alpha$ Cen A and B and HD 80809, have been added to the monitoring list. Both 61 Cyg A and HD 80809 are stars with known calcium cycles, and both systems have been monitored in $\mathrm{Ca} \mathrm{H}$ and $\mathrm{K}$ for a significant amount of time, while, unfortunately, no systematic longterm calcium monitoring is available for the $\alpha$ Cen system. The results of these monitoring observations have been published by Hempelmann et al. (2006), Robrade et al. (2007), Favata et al. (2004), Favata et al. (2008) and Robrade et al. (2012); as an example I show in Fig. 6 the XMM-Newton lightcurve of $61 \mathrm{Cyg}$ A, covering the time frame from 2002 (when the 61 Cyg system became visible to XMM-Newton) until now. According to Baliunas et al. (1995) the calcium cycle of 
this star is 7 years, and from Fig. 7 it is obvious and also in some ways expected that the X-ray emission from 61 Cyg A follows the Ca emission very well. A similar dependence between calcium and X-ray cycles is found for HD 80809, which is a little surprising since its X-ray luminosity is much larger than that of 61 Cyg A. Similarly, an X-ray cycle may exist for the case of $\alpha$ Cen A, however, since no calcium data is available for that star, it is too early to draw any firm conclusions on a possible X-ray cycle.

In this context it is surprising that variations as shown in Fig.7 can be measured at all. Naively one would assume that short term variability in the form of flares and rotational modulation would actually destroy the usually much smaller variability due to cyclic emission. Also it has to be kept in mind that the X-ray observations are by necessity snapshot observations, taken on a much shorter timescale than the rotational variation, while calcium data are often averaged over a whole rotational period. In actual fact, the individual XMM-Newton pointings of $61 \mathrm{Cyg} \mathrm{A}$ and B last typically $10 \mathrm{ksec}$. An examination of the whole data set available for 61 Cyg shows that, indeed, flares do occur, fortunately on a time scale shorter than $10 \mathrm{ksec}$ and therefore "quiescent" emission periods with the corresponding emission levels can always be established, and it is these quiescent emission intervals that show cyclic variability (Robrade et al. 2012).

\section{The extended solar minimum between 2008 - 2009}

The last solar minimum in the years 2008 and 2009 received a lot of attention, both from the science community and the general public, because of the prolonged observed absence of sunspots. This minimum reminded of the Maunder-Minimum between 1650 - 1715, which is also characterized by an almost complete the absence of sunspots (cf., Fig. 1). Since the Maunder minimum period coincides with the time of the "little ice age" (cf., Eddy 1976), there is considerable interest in the question what the properties of the solar chromosphere and corona were at that time.

In the framework of the Mount Wilson Ca HK monitoring program the Sun has been regularly observed and its S-index was determined just like the S-indices of all other program stars (cf., Baliunas et al. (1995)). A very clear cycle is found in these data, in fact, the cycle of the Sun appears to be one of the best cycles (classified as "excellent" by Baliunas et al. (1995)) in the whole list of cycles presented in the paper by Baliunas et al. (1995). With our Hamburg Robotic Telescope (HRT, Hempelmann et al. 2005) we observed solar calcium spectra in the time frame between August 2008 to July 2009. Both sky spectra as well as Moon spectra were used and disk-averaged S-indices derived in the usual way. In Fig. 7 I display the resulting S-index "light" curve, taken from Mittag (2010). Interestingly but not surprisingly, the obtained S-values are consistently
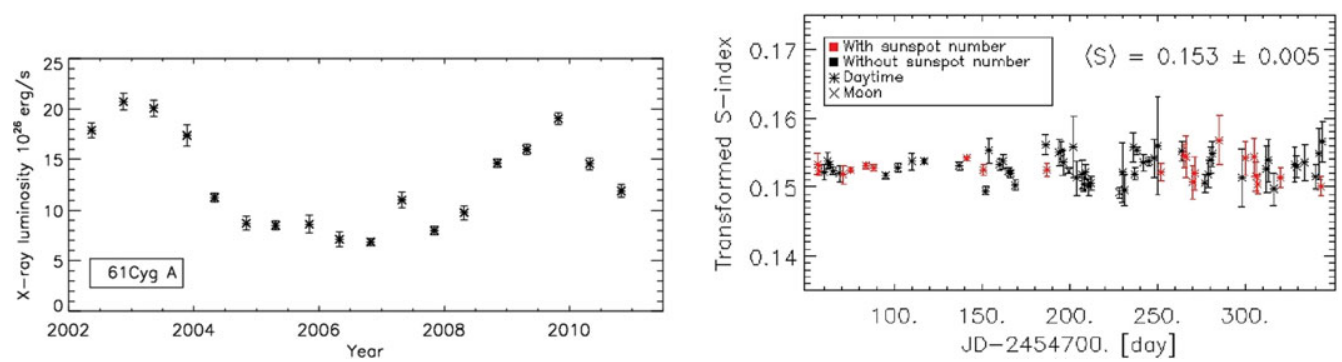

Figure 7. Left panel: XMM-Newton light curve of 61 Cyg A (taken from Robrade et al. (2012). Right panel: solar S-index variation in 2008-2009 (taken from Mittage 2010). For a colour figure, see the online version of this paper. 
low, at times the $\mathrm{S}$-index drops even to below $\mathrm{S}=0.15$ and the average $\mathrm{S}$-index value $(\mathrm{S}=0.153)$ in the observed period is below the minimum observed by Baliunas et al. (1995), i.e, $\mathrm{S}=0.16$; note the recorded S-indices were taken from both day-time spectra as well as lunar spectra and have been corrected for the effects of scattering. An inspection of contemporaneously obtained solar $\mathrm{Ca} \mathrm{K}$ images also shows that plage regions were largely absent from the Sun during that period, and there are days without any clear signature of plage emission at all. We, therefore, conclude that not only was the sun spot number very low, but also the solar S-index was below the values measured between 1950 to 1990 and presented by Baliunas et al. (1995).

Is this observed low state of the Sun then already the state of the Sun during the Maunder minimum? There has been considerable debate in the literature on the definition of Maunder-Minimum stars and a recipe on how to find such objects. I do not want to delve into that discussion (see Saar 2012), I simply remark that, on the one hand, claims were published that the fraction of Maunder-Minimum stars, for example in the Mount Wilson sample, is quite substantial (Baliunas \& Jastrow 1990), while, on the other hand, it was argued by other authors (cf., Wright 2004) that Maunder-Minimum stars in the Mount Wilson sample might simply not exist and have in fact been mistaken for subgiants. To clarify the issue and settle the position of the Sun within the stars, we used the catalog of Ca HK measurements published by Duncan et al. (1991), selected those stars with precise parallax information in order to classify the stars into main sequence stars, subgiants and giants, and study their distribution in an S-index vs. $M_{V}$ diagram (cf., Fig.8); a far more extended discussion of this will be presented by Mittag et al. (2012). Fig.8 demonstrates that, indeed, subgiants and giants can have significantly smaller S-indices than their main sequence brothers, and therefore subgiants and giants, erroneously interpreted as main sequence objects, will result in make-believe Maunder minimum stars as pointed out by Wright (2004). However, as also apparent in Fig.8 and extensively discussed by Mittag et al. (2012), there is still a very well defined lower envelope in S-index, which empirically defines the lowest observed chromospheric activity state of a main sequence star. For solar-like stars with $B-V 0.64$ this lower envelope to the observed S-index distribution is around $S_{\min } 0.145$, and it is therefore clear that during its extended minimum between 2008 - 2009 the Sun came - in terms of S-index - very close to this empirically observed bottom of the S-index distribution for main sequence stars. In other words, the activity of the Sun as observed in Ca HK was essentially as low as solar-like stars at the effective temperature of the Sun can ever get, and therefore it is suggestive to interpret the minimum Sun between 2008 - 2009 as a close-up example of a Maunder minimum star.

\section{A minimum X-ray flux for the Sun and the stars?}

While long-term solar monitoring at soft X-ray wavelengths is not available yet, significant efforts have been undertaken in establishing solar reference spectra over a wide spectral range in order to facilitate a variety of studies of the Earth's climate and how it might be affected by the solar output in different energy bands. Woods et al. (2009) published Solar Irradiance Reference Spectra (SIRS) for the so-called Whole Heliospheric Interval (WHI) in Carrington Rotation 2068 covering the time span between March 20 to April 16 2008. In that time frame solar activity was already very low and I specifically use the data set from April 10 - April 16, where the Sun's activity was already very much depressed with rather low sun spot numbers and very low $10.7 \mathrm{~cm}$ radio fluxes and therefore should - as pointed out by Woods et al. (2009) - represent the solar minimum 
conditions very well. For reference purposes I plot the solar surface flux in cgs units (per bin) for the WHI quiescent spectrum in Fig. 8.

I then proceed to compute integrated solar X-ray surface fluxes in various pass bands. Since the various X-ray missions tend to use their own specific band passes, I compute the X-ray fluxes in pass bands ranging from some low energy threshold (LET in keV) up to $2.4 \mathrm{keV}$; note the 'official' ROSAT band pass is $0.1-2.4 \mathrm{keV}$. The precise choice of the upper energy threshold is immaterial in this context since the solar input spectrum is very soft and contains very little flux at wavelengths below $10 \AA$ (cf., Fig.9). As is clear from Fig.9, the choice of the lower energy threshold is crucial for the resulting surface flux value. The formal value of surface flux in the ROSAT $0.1-2.4 \mathrm{keV}$ band for the WHI spectrum is $9062 \mathrm{erg} / \mathrm{cm}^{2} / \mathrm{s}$, which is very close to the estimated value of $10^{4} \mathrm{erg} / \mathrm{cm}^{2} / \mathrm{s}$ as the minimum X-ray surface flux for stars. Had the Einstein Observatory band pass of 0.2 - $4 \mathrm{keV}$ band pass been chosen, a much smaller surface flux value of $3762 \mathrm{erg} / \mathrm{cm}^{2} / \mathrm{s}$ would have resulted.

It is further useful to compare the resultant X-ray surface fluxes with previously published "old" values. Specifically I use the Skylab studies by Maxson \& Vaiana (1977) as well as the YOHKOH SXT studies by Hara et al. (1994). Maxson \& Vaiana (1977) produce (in their Fig. 10) a line of sight emission measure curve for coronal holes $(\mathrm{CH})$ as well as an emission measure for large scale quiescent structure (LSS), while Hara et al. (1994) produce a two-temperature fit to their filter ratio data. Using CHIANTI, I calculate the equivalent soft X-ray fluxes in the 0.1-2.4 keV pass band, which are plotted in Fig.10; as is clear from Fig.10, both the Skylab and YOHKOH SXT data yield values lower than the SIRS, which is, however, not surprising since the YOHKOH SXT specifically refers to a coronal hole. On the other hand, all numbers agree reasonably well and do seem to point at the existence of a minimum X-ray flux also for the Sun. Converting the SIRS surface flux of $9062 \mathrm{erg} / \mathrm{cm}^{2} / \mathrm{s}$ to a global X-ray luminosity, I find $\mathrm{L}_{X} \approx 5.5 \times$ $10^{26} \mathrm{erg} / \mathrm{sec}$, which I propose to use as a minimal solar X-ray luminosity.

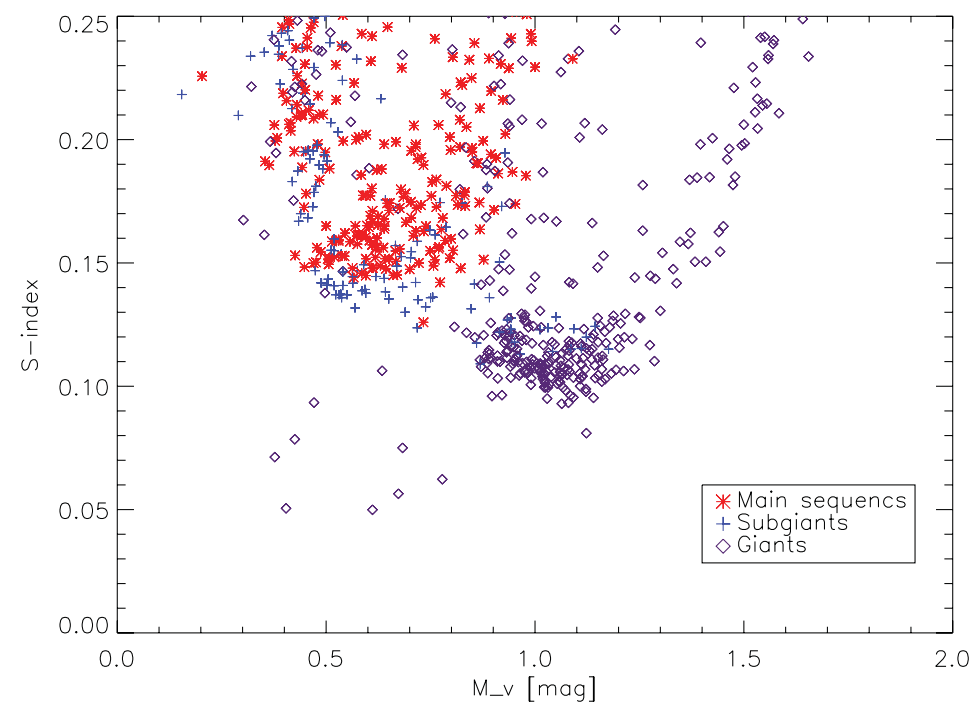

Figure 8. S-index (from Duncan et al. (1991)) for main sequence stars (asterisk), subgiants (plusses) and giants (diamonds), courtesy M. Mittag. For a colour figure, see the online version of this paper. 

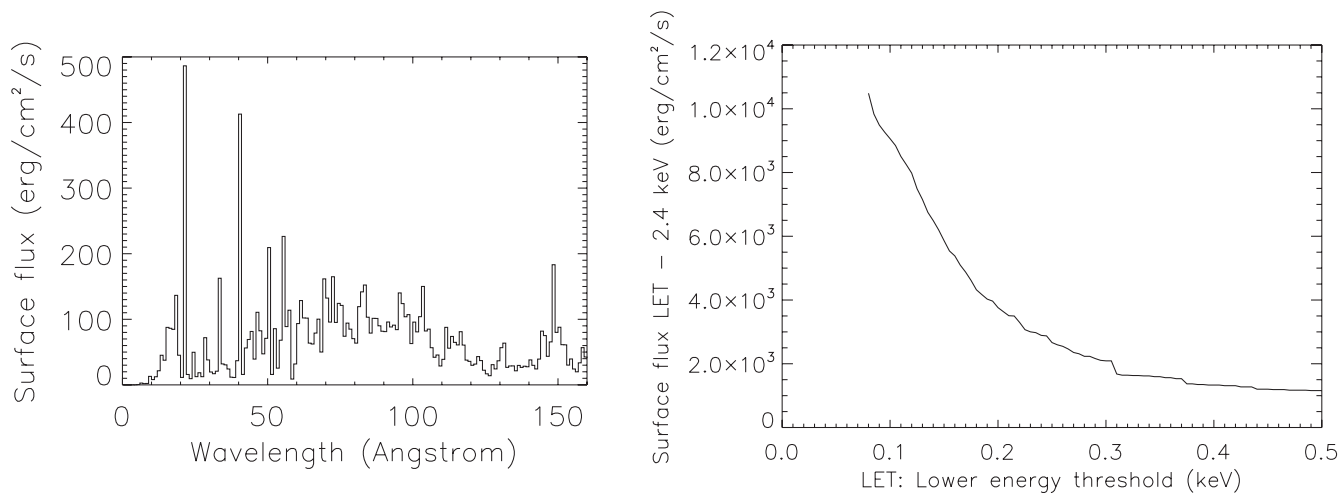

Figure 9. Left panel: solar surface flux (in cgs units per bin) vs. wavelength from SIRS. Right panel: X-ray surface flux as a function of passband; the ordinate is the lower energy threshold (see text for details).

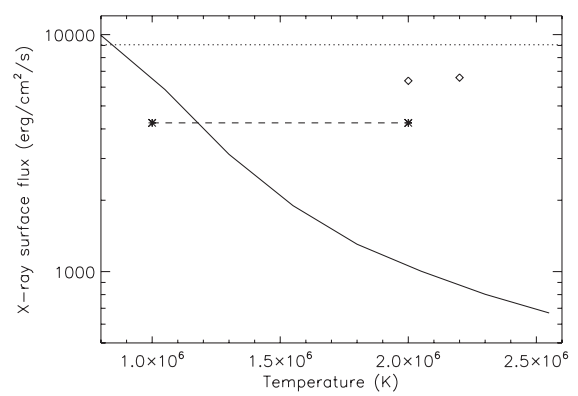

Figure 10. Comparison of calculated solar surface flux values: dotted line (SIRS), solid line represents the $\mathrm{CH}$ emission measure from Maxson \& Vaiana (1977), diamonds correspond to LSS from Maxson \& Vaiana (1977), and two-temperature CH from Hara et al. (1994).

The close agreement between the minimum solar flux and the lower envelope of the stellar flux distribution is certainly somewhat fortuitous since there is considerable uncertainty in converting the measured PSPC count rates to X-ray surface fluxes. This conversion depends, first, on the X-ray temperature (the precise value of which is not really known for these soft sources), and second, it depends on stellar size and distance. Therefore the scatter of data points near the lower flux threshold is not really surprising, rather, the real surprise is that the minimum flux corona as represented in the WHI spectrum fits so well to the minimum X-ray fluxes of stars. This agreement leads me then to argue that the X-ray corona of the Sun as observed in the extended last solar minimum is, first, compatible with the minimal X-ray coronae we observe on other stars and, second, that it is very likely to be compatible with the corona of the Sun during the time of the Maunder minimum. This view is consistent with the findings of Eddy (1976), who discusses reports on coronal sightings during total solar eclipses observed during the Maunder minimum period and finds that the solar wind was probably a fast wind and that the corona visible during the eclipse was by comparison small and unstructured, but still present.

\section{References}

Baliunas, S. \& Jastrow, R. 1990, Nature, 348, 520

Baliunas, S. L., Donahue, R. A., Soon, W. H., et al. 1995, Ap. J., 438, 269 
Duncan, D. K., Vaughan, A. H., Wilson, O. C., et al. 1991, Ap. J. Supp., 76, 383

Eddy, J. A. 1976, Science, 192, 118

Edlén, B. 1945, MNRAS, 105, 323

Favata, F., Micela, G., Baliunas, S. L. et al. 2004, Astron. Ap., 418, L13

Favata, F., Micela, G., Orlando, S. et al. 2008, Astron. Ap., 490, 1121

Foukal, P., Fröhlich, C., Spruit, H., \& Wigley, T. M. L. 2006, Nature, 443, 161

Hara, H., Tsuneta, S., Acton, L. W. et al. 1994, PASJ, 46, 493

Hempelmann, A., Schmitt, J. H. M. M., \& Stȩpień, K. 1996, Astron. Ap., 305, 284

Hempelmann, A., Schmitt, J. H. M. M., Baliunas, S. L., \& Donahue, R. A. 2003, Astron. Ap., 406, L39

Hempelmann, A., Gonzalez Perez, J. N., Schmitt, J. H. M. M., \& Hagen, H. J. 2005, 13th Cambridge Workshop on Cool Stars, Stellar Systems and the Sun, 560, 643

Hempelmann, A., Robrade, J., Schmitt, J. H. M. M., et al. 2006, Astron. Ap., 460, 261

Maxson, C. W. \& Vaiana, G. S. 1977, Ap. J., 215, 919

Mittag M., 2010, PhD Thesis available in electronic form at "http://www.hs.unihamburg.de/DE/Ins/HRT/hrtpublication.html"

Mittag, M. 2012, in preparation

Pevtsov, A. A., Fisher, G. H., Acton, L. W., et al. 2003, Ap. J., 598, 1387

Robrade, J., Schmitt, J. H. M. M., \& Hempelmann, A. 2007, Memorie della Societá Astronomica Italiana, 78,311

Robrade, J. 2012, in preparation

Saar, S. 2012, these proceedings

Schmitt, J. H. M. M., Golub, L., Harnden, F. R., Jr., et al. 1985, Ap. J., 290, 307

Schmitt, J. H. M. M. 1997, Astron. Ap., 318, 215

Schröder, C. \& Schmitt, J. H. M. M. 2007, Astron. Ap., 475, 677

Stern, R. A., Alexander, D., \& Acton, L. W. 2003, The Future of Cool-Star Astrophysics: 12th Cambridge Workshop on Cool Stars, Stellar Systems, and the Sun, 12, 906

Svalgaard, L. 2012, these proceedings

Vaiana, G. S., Cassinelli, J. P., Fabbiano, G. et al. 1981, Ap. J., 245, 163

Woods, T. N., Chamberlin, P. C., Harder, J. W. et al. 2009, Geophys.Res.Letters J., 360, 1101

Wright, J. T. 2004, A.J., 128, 1273

\section{Discussion}

MARK GIAMPAPA: Images of SOHO for the Sun show bright maximum and nothing at the minimum. Can you explain why the peak to peak excursion for other stars are much smaller than for the Sun?

JÜRGEN SCHMiTT: The measured peak-to-peak variations over a cycle depend sensitively on the spectral range that is used. This also applies to solar data, for example the GOES hard X-ray fluxes. In the stellar case one is dominated by cooler plasma which is ensuing smaller variations.

JEFFREY LINSKY: With the extended VLA we observe many radio stars with different emission mechanism, gyro-resonance or coherent emission, not thermal. Can you comment on that?

JÜRGEN SCHMITT: I realize that the sensitivity of radio observations has been improved. It would of course be extremely valuable to have a stellar analog to the $10.7 \mathrm{~cm}$ radio flux monitoring that provides an excellent diagnosis for the solar cycle. 